\title{
The Applicability of Incoherent Array Processing to IMS Seismic Arrays
}

Steven J. Gibbons ${ }^{1}$

${ }^{1}$ NORSAR, Kjeller, Norway

Contact: S. J. Gibbons, NORSAR, P.O. Box 53, 2027 Kjeller, Norway. (steven@norsar.no; Tel: $+4763805939)$ 
Abstract - The seismic arrays of the International Monitoring System (IMS) for the Comprehensive Nuclear-Test-Ban Treaty (CTBT) are highly diverse in size and configuration with apertures ranging from under $1 \mathrm{~km}$ to over $60 \mathrm{~km}$. Large and medium aperture arrays with large inter-site spacings complicate the detection and estimation of high frequency phases lacking coherence between sensors. Pipeline detection algorithms often miss such phases since they only consider frequencies low enough to allow coherent array processing, and phases that are detected are often attributed qualitatively incorrect backazimuth and slowness estimates. This can result in missed events due to either a lack of contributing phases or by corruption of event hypotheses by spurious detections. It has been demonstrated previously that continuous spectral estimation can both detect and estimate phases on the largest aperture arrays, with arrivals identified as local maxima on beams of transformed spectrograms. The estimation procedure in effect measures group velocity rather than phase velocity, as is the case for classical f-k analysis, and the ability to estimate slowness vectors requires sufficiently large inter-sensor distances to resolve time-delays between pulses with a period of the order 4 to 5 seconds. Spectrogram beampacking works well on five IMS arrays with apertures over 20 km (NOA, AKASG, YKA, WRA, and KURK) without additional post-processing. Seven arrays with 10 to $20 \mathrm{~km}$ aperture (MJAR, ESDC, ILAR, KSRS, CMAR, ASAR, and EKA) can provide robust parameter estimates subject to a smoothing of the resulting slowness grids, most effectively achieved by convolving the measured slowness grids with the array response function for a 4 or 5 second period signal. Even for medium aperture arrays which can provide high quality coherent slowness estimates, a complementary spectrogram beampacking procedure could act as a quality control by providing non-aliased estimates when the coherent slowness grids display significant sidelobes. The detection part of the algorithm is applicable to all IMS arrays, with spectrogram-based processing offering a potential reduction in the false alarm rate for high frequency signals. Significantly, the local maxima of the scalar functions derived from the transformed spectrogram beams are robust estimates of the signal onset time. High frequency energy is of greater importance for lower event magnitudes and in the cavity decoupling detection evasion scenario. There is a need to characterize both propagation paths with low attenuation of high frequency energy and situations in which parameter estimation on array stations fails.

Key words: Seismic arrays, nuclear explosion monitoring, CTBT, signal incoherence, parameter 
estimation, spectral estimation

\section{Introduction}

The International Monitoring System (IMS) for monitoring compliance with the Comprehensive Nuclear-Test-Ban Treaty (CTBT) consists of global networks of seismic, infrasonic, hydroacoustic and radionuclide sensors for detecting potential violations of the treaty (see DAHLMAN et al., 2009). The seismic network consists of both 3-component stations (at single sites) and seismic arrays (deployments of multiple sensors at separate sites over a limited geographical region). The task of any seismic station in the global network is to detect phase arrivals generated by any seismic disturbance and attribute parameters such that signal detections from different stations can be associated to form event hypotheses in an automatic seismic bulletin (e.g. RINGDAL and KVÆrNA, 1989). The most important parameter in the phase association process is the arrival time. However, the effectiveness of an association algorithm will also depend upon the reliability of the estimates for two basic parameters: the apparent velocity, $v_{\text {app }}$, from which a phase identification is usually made, and the backazimuth, BAZ, the direction from which the phase arrives. A highly erroneous, or qualitatively incorrect, parameter estimate is likely to prevent the detection from being associated with the correct event hypothesis and may result in the non-detection of a seismic event.

The ability to detect and classify seismic phases is generally superior for array stations than for 3-component stations (e.g. KVÆRNA et al., 2011) since the stack-and-delay beamforming can improve significantly the signal-to-noise-ratio (SNR), and the time-delay estimation methods for slowness vectors are typically more robust and more widely applicable than methods based on the polarization of particle motion (see e.g. KVÆRNA and RINGDAL, 1992). Reviews of array processing techniques for phase detection and parameter estimation are given by, for example, SCHWEITZER et al. (2002); Rost and Thomas (2002, 2009). However, the classical concept of array processing breaks down when coherence between sensors of an array is lost (e.g. Bungum and HusEBYE, 1971)

and an array for low frequency seismic waves becomes, in effect, a network for higher frequencies (Levander and Nolet, 2005). In these cases we need to look to incoherent methods, or network techniques, to detect and make useful parameter estimates of seismic arrivals.

The IMS seismic arrays are highly heterogeneous in terms of the geometry and the number 
of sensors (e.g. Douglas, 2002). The configurations of all primary and auxiliary IMS arrays, with the exception of the so-called medium period arrays, providing data as of September 2011 are displayed in Figure 1. Over an order of magnitude separates the array apertures between the smallest arrays (aperture typically under $4 \mathrm{~km}$ ) and the largest (aperture up to $70 \mathrm{~km}$ ). The reason for the dissimilarity between the different array stations is that they were built at different times to be best suited to differing monitoring priorities. In contrast, the IMS infrasound arrays (CHRISTIE and CAmpus, 2010) have been deployed over a period of approximately one decade with the aim of comprising a unified network, and there is far less variability in the array apertures and sensor configurations.

Many of the larger arrays are so-called legacy stations dating back to the 1960s and 1970s, which were designed to provide optimal detection and estimation for teleseismic P-phases with energy concentrated at around $1 \mathrm{~Hz}$. Figure 2 displays signals from two underground nuclear explosions at the Semipalatinsk test site in the former Soviet Union recorded at the NORSAR array (now labelled NOA) at a distance of 37.8 degrees. One of the events is significantly smaller than the other with an approximate factor of 10 separating the maximum amplitudes in the frequency bands where the signal exceeds the noise level significantly. It is clear that there is significant energy at frequencies well above $1 \mathrm{~Hz}$, and that it is the SNR at the lower frequencies which is most greatly reduced given an event of smaller size. The SNR above $4 \mathrm{~Hz}$ is remarkable both due to the large epicentral distance and the use of an analogue anti-aliassing filter with a cut-off at $4.5 \mathrm{~Hz}$.

Figure 3 illustrates the result of beamforming over the large aperture NOA array for the two events displayed in Figure 2. The top left panel shows the signals from the larger event, filtered 0.5$1.5 \mathrm{~Hz}$, on three sensors from each of two different subarrays, together with a beam constructed using carefully calibrated time-delays. The signal amplitudes on the NC6 subarray are significantly greater than the amplitudes on the NB2 subarray (c.f. Ringdal and HuseBye, 1982) and the amplitude of the signal on the resulting beam is significantly lower than the greatest signal amplitudes recorded on some single sensors. However, the beamforming suppresses the preceding noise sufficiently to ensure an improved SNR on the array beam (this is especially clear for the smaller event in the upper right panel). The SNR in the $0.5-1.5 \mathrm{~Hz}$ band essentially does not exceed unity for the single channels, although the signal is clearly visible on the beam. The lower panels in Figure 3 are the 
corresponding displays for the 4-8 Hz band. The single-channel SNR is considerably better for all traces, but the waveform dissimilarity between the different sensors is now evident and the beam results in significant signal degradation.

This example illustrates a fundamental problem in array processing: that moving to higher frequencies improves the single-channel SNR at the expense of signal coherency and, consequently, the ability to perform classical array processing. It was pointed out by RINGDAL et al. (1975) that incoherent (waveform envelope) methods were often necessary to exploit this higher frequency energy on the NORSAR array. The evident limitations of the very large aperture arrays resulted in the development of far smaller arrays capable of processing signals at much higher frequency, in particular for phases from events at regional distances (e.g. MYkKeltveit et al., 1990). The current IMS, including many small and medium aperture arrays, is closer to the global system for monitoring nuclear explosions envisaged in the 1950s (ROMNEY, 1985) than the sparse network of very large aperture arrays developed in the 1960s and 1970s.

There is no such thing as a universal seismic array. An array with a sufficiently large aperture, and sufficiently large inter-site spacings, to provide sharp resolution and good noise reduction for teleseismic phases is likely to be unable to process high frequency regional phases using classical beamforming. Similarly, small-aperture arrays with geometries that are optimized for the detection and characterization of high frequency regional phases are likely to provide poor resolution and low SNR gain for teleseismic phases. Special processing is necessary to improve the detection and estimation of teleseismic phases on small aperture arrays amid strong and coherent noise (SELBY, 2011). Similarly, complementary processing is necessary to detect and resolve phases that are incoherent over very large aperture arrays. The purpose of this paper is to explore the applicability of the spectrogram beamforming method of GiBBons et al. (2008) to different arrays in the IMS network, in particular to arrays where it is demonstrated that classical array processing fails for high frequency regional phases. We examine the anticipated performance of the method as a function of array aperture, discuss the application of recent innovations in signal processing to improving the performance, and discuss the options available for situations in which the method is likely to fail. 


\section{Detection and Parameter Estimation on Seismic Arrays Using Continuous Spectral Estimation}

In the most common framework for seismic phase detection, a single trace is bandpass filtered in a frequency band deemed to be optimal for the anticipated phase. This single trace may be the output from a single sensor or a beam constructed from the output of many sensors of an array with appropriate time delays applied. Detections are then declared when the signal-to-noiseratio (SNR), i.e. the ratio between the short-term-average (STA) and the long-term-average (LTA), exceeds a specified threshold. See, for example, WithERs et al. (1998) and references therein for a summary of such detection algorithms. Alternatives to such procedures may operate on 2dimensional representations of the seismogram, with time on one axis and frequency or scale on the other, to identify amplitude changes characteristic of anticipated phase arrivals or full wavetrains.

Wavelet transform methods (e.g. YomoGIDA, 1994; OonincX, 1999; Gendron et al., 2000; ZHANG et al., 2003; CAPIlla, 2006) provide coefficients for wavelets of different scales from a selected basis function, and phase detection algorithms can trigger on a significant increase in the size of wavelet coefficients over a specified range of scales. Alternatively, the spectrogram or sonogram provides signal amplitude over a uniform discretization of time and frequency. Seismic signal detection on spectrograms has traditionally focused on pattern recognition and identification of characteristic time/frequency signatures of seismic events (e.g. Joswig, 1990, 1995; Ḧ̈GE and JoswiG, 2009). Recently, TAYLOR et al. (2010) demonstrated a procedure for detecting transient, short-duration, signals on spectrograms using scan statistics. The aim was to detect signals from events at local distances which frequently evaded detection by classical procedures due to a complicated background noise field. They pointed out that a large bandwidth signal would register over a greater number of elements in spectrogram columns than noise transients, such that a 2-D linear filter would emphasize columns of the spectrograms that were relatively rich in energy. The resulting detection procedure was demonstrated to have a lower false alarm rate than a corresponding STA/LTA algorithm.

GibBons et al. (2008) describe an application of continuous spectral estimation to solving the detection and parameter estimation problem for incoherent signals on large aperture seismic arrays. Figure 4 displays a typical example of such a signal. The event displayed is included in the Reviewed 
Event Bulletin (REB) of the International Data Center (IDC) and both Pn and Sn phase arrivals are clearly identifyable on the filtered waveforms on the WRA array (Warramunga, Australia). WRA is an exceptionally quiet station with a superb detection capability for signals from events at teleseismic distances. However, the large array aperture and inter-site spacings which are so favourable for teleseismic array processing make the array almost unusable for events at regional distances. Despite an epicentral distance of around $700 \mathrm{~km}$, high frequency energy dominates and both $\mathrm{P}$ and $\mathrm{S}$ phases are not visible above the background noise below $2 \mathrm{~Hz}$. The velocity and azimuth for the Pn phase reported in the REB are consistent with the phase type and direction, although it is clear from the lower-left f-k plot in Figure 4 that the relative power for this slowness vector is only marginally greater than that for other very different slowness vectors. The Sn phase in the REB is associated with a velocity and azimuth that are qualitatively incorrect and the lower-right f-k plot in Figure 4 makes clear that there are no obvious candidate directions to choose from. The incoherence problem is frequently mitigated by removing traces recorded on more distant sensors from the calculation or by selecting a lower frequency band. In this case, it is not possible to use a lower frequency band due to SNR considerations. No frequency band or subset of sensors was found that provided a qualitatively correct slowness estimate for this Sn phase on WRA.

Figure 5 illustrates the steps that the algorithm of GiBBons et al. (2008) takes to detect and estimate propagation parameters for such phases. One of very few processing parameters in the procedure is $L$, the length of the window used for spectral estimation. The window must be long enough to allow a robust estimate of the energy at each of the frequencies of interest (typically 2 to $15 \mathrm{~Hz}$ for regional phases), the multitaper method of Thomson (1982) being deemed ideal for spectral estimation on the short-duration data segments. $L$ must not be so small that the continuous spectral estimates are over-sensitive to rapidly evolving properties of the waveforms (that are likely to vary significantly from sensor to sensor), but must neither be so large that energy is smeared out excessively, diminishing the likelihood of being able to estimate a time-delay between arrivals on neighbouring sensors. GibBons et al. (2008) found empirically that windows between 2.8 and 3.8 seconds long provided a satisfactory balance between robustness and resolution, and the spectrograms displayed in traces 4-6 of Figure 5 are calculated using windows of length 3.2 seconds under the numerical implementation of PRIETo et al. (2009).

Draft

Pure appl. Geophys.

September 29, 2012 
Unlike the transient signals considered by TAYLOR et al. (2010), which appear as vertical lines on the spectrograms, the arrivals in Figure 5 are followed by coda with amplitude greater than or equal to that of the direct wavefronts, resulting in spectrograms that resemble more closely smoothed stepfunctions in time than the desired smoothed delta functions. GibBons et al. (2008) appreciated the need to apply a transformation to the spectrograms to make them more comparable to the vertical line spectrograms, with local maxima occurring as close as possible to the phase arrival time. If $A(f)_{t+}=A(f, t, L)$ denotes the amplitude density spectrum measured for the window immediately following a time $t$, and $A(f)_{t-}=A(f, t-L, L)$ the spectral estimate in the window ending at time $t$, then the transformed spectrogram denoted

$$
S(f, t)=\left(\log _{10}\left[A(f)_{t+}\right]-\log _{10}\left[A(f)_{t-}\right]\right) \log _{10}\left[A(f)_{t+}\right]
$$

provides local maxima at times characterized by both a relatively high amplitude and an increase in amplitude. Such transformed spectrograms are displayed in traces 7-9 of Figure 5. As pointed out by TAYLOR et al. (2010), the times of the direct phase arrivals are characterized by high values over a broad band of frequencies whereas noise peaks are localized at rather random frequencies. Local maxima in the transformed spectrograms resulting from noise bursts tend to be suppressed under the spectrogram beamforming operation, whereas the local maxima from the direct phases interfere constructively as the transformed spectrograms are stacked with appropriate time-delays. The beamforming process itself, together with the detection of local maxima in the spectrogram beams, is covered in detail by GiBBons et al. (2008).

The two slowness grids in Figure 5 cover the same parameter space as the f-k spectra in Figure 4, but display maximum values of spectrogram beams formed in the time domain, averaged over frequencies, rather than relative beam power calculated in the frequency domain. These so-called beampacking grids indicate that a qualitatively correct slowness estimate is obtained for both the Warramunga Pn and Sn phases. Since it is evident from Figure 4 that a correct classification of these relatively high frequency phases is either impossible or subject to an unacceptably high likelihood of failure, it is recommended that both forms of estimation are carried out: coherent f-k analysis for high resolution slowness estimates of lower frequency teleseismic phases and incoherent processing for detection and characterization of regional phases.

The regional signals from which these estimates are made contain essentially no energy above the Draft Pure appl. Geophys.

September 29, 2012 
noise level at frequencies below $2 \mathrm{~Hz}$. The transformation applied to the spectrograms of the single channels generates functions which resemble lower frequency arrivals and the estimation problem shifts from one of measuring a phase velocity to one of measuring a group velocity. The period of the scalar functions generated by the process described will depend both upon $L$, the length of the data window used for spectral estimation, and on how emergent a given signal is. Whether or not the spectrogram beamforming method can be used to estimate a slowness vector on a given seismic array becomes a question of whether or not the distances between sensors are great enough for resolution of these relatively long-period functions. Given $L$ between 3 and 4 seconds as displayed here, the scalar functions obtained from the transformed spectrograms have a typical frequency of about 0.20 to $0.25 \mathrm{~Hz}$. Five IMS arrays, NOA, AKASG, YKA, KURK, and WRA, have apertures of the order of $20 \mathrm{~km}$ or more and incoherent array processing on each of these arrays is likely to produce results of a similar quality to those displayed in Figure 5.

\section{Improving Incoherent Slowness Estimates by Smoothing with the Array Response Function}

The incoherent slowness vector estimates displayed in Figure 5 indicate both apparent velocities which would identify the phase correctly and backazimuth estimates that are sufficiently consistent for any detection association algorithm to use both phases in an event hypothesis. However, an inspection of the slowness grids identifies a cause for concern. The regions of parameter space for which the spectrogram beam maxima are consistent with wavefront hypotheses are very broad. Whereas it appears that the correct quadrant of slowness space is likely to be selected, the risk of choosing a qualitatively incorrect estimate is high. Since the waveforms are incoherent, the transformed spectrograms will also differ from site to site. Although there will be more uniformity in the spectrograms than in the phase structure of the underlying waveforms, local maxima appear in the beampacking grids which are not predicted by the (plane wavefront) propagation model. Inspection of the slowness grids using the human eye identifies credibility contours with a significant degree of symmetry, which may suggest a different slowness vector to that inferred from consideration of the beam maximum alone.

Draft

Pure appl. Geophys.

September 29, 2012 
The form of the full f-k spectrum has been considered in numerous previous studies to resolve ambiguity in, or improve the resolution of, slowness vector estimates. KENNETT et al. (2003) compared the measured $\mathrm{f}-\mathrm{k}$ spectrum with the corresponding theoretical array response function (ARF) at different frequencies, in order to select the most plausible slowness vector using a Neighbourhood Algorithm. The primary application of this procedure was to mitigate the effects of spatial aliassing when a high frequency signal was observed on arrays with relatively sparse sensors. NisHIDA et al. (2008) performed $\mathrm{f}-\mathrm{k}$ analysis of long period seismic data and noted that the slowness resolution varied greatly in different directions due to the elongated geometry of the array (in this case a Southwest to Northeast network covering Japan). They noted that this difficulty could be mitigated by performing a deconvolution of the observed f-k spectrum with the ARF. The improvement to parameter estimates resulting from sharpening of $\mathrm{f}-\mathrm{k}$ spectra by deconvolution with the ARF was demonstrated further by PicOzzI et al. (2010). The problem we are addressing is fortunately significantly simpler and can be addressed by finding the translation of the ARF which provides the best match with the observed slowness grid. This amounts to a smoothing of the slowness grid with the ARF which will reduce the influence of the departures from the propagation model.

Figure 6 (a) displays a slowness grid from a spectrogram beamforming procedure for a high frequency regional phase on the Kurchatov array (KURK) in Kazakhstan. A qualitatively correct apparent velocity has been measured, i.e. one consistent with a regional P-type phase, although it is clear that the loci of slowness vectors that are candidates for the optimal array-gain are quite widely spread over slowness space. The ARF for the time-domain spectrogram beampacking procedure is not well defined in the same way that it is for $\mathrm{f}-\mathrm{k}$ analysis at a given frequency (e.g. Schweitzer et al., 2002; Kennett, 2002). However, in Figure 6 (b), we estimate an approximation of an empirical ARF by simply taking the scaled spectrogram from one array element, duplicating it over every sensor of the array, and then measuring the array-gain over the slowness space by stacking the identical channels with the appropriate time-delays. The ARF is approximately straight-edged with the same orientation as the axes of the cross-array geometry (see Figure 1). A correlation between two time-series, $f(t)$ and $g(t)$, is equivalent to convolving the first time-series with a time-reversed copy of the second time-series, $g(\tau-t)$. This is most effectively computed by taking the inverse Fourier transform of the product of the Fourier transforms of $f(t)$ and $g(\tau-t)$. Similarly, taking 
Fourier transforms in two-dimensions of the slowness grids in panels (a) and (b) of Figure 6 allows a rapid frequency domain calculation of the convolution between the two grids which describes, as a function of the horizontal slowness parameters, the quality of the match between the measured slowness grid and the ARF.

The resulting function, displayed in Figure 6 (c), also covers a broad region of slowness space, but has a single, central, local maximum. The smoothing procedure illustrated in Figure 6 (c) has been applied to many spectrogram phase detections on the five largest IMS seismic arrays and appears to improve the stability of slowness estimates made using this procedure. While an empirical ARF as displayed in Figure 6 (b) can be calculated, such functions almost always resemble very closely the array response for a 4 second sinusoid and the output from the smoothing process differed rarely when such a function was used as the ARF. A very impulsive signal may result in a faster varying transformed spectrogram, and a highly emergent signal may result in a more slowly varying function, but this should affect only the spread and not the shape of the ARF. We propose that the resolution in slowness space of the spectrogram beampacking procedure is well estimated using a 4 second ARF, and that such a function can be used to estimate the applicability of the procedure over arrays of different aperture.

\section{The Applicability of Incoherent Parameter Estimation as a Function of Array Aperture}

Figure 7 displays the ARF for a signal with period 4 seconds for six IMS arrays with different apertures, all of which have difficulties in processing signals from events at regional distances. As demonstrated by GiBBons et al. (2008), the resolution provided by the NOA array (with aperture of over $60 \mathrm{~km}$ ) is high enough to provide unambiguous slowness estimates for any regional phases without the need for a smoothing in the slowness grid (Figure 7 a). The AKASG array (Malin, Ukraine) is the second largest array in the IMS but, with an aperture significantly smaller than that of the NOA array, has a significantly broader ARF (Figure 7 b). The ellipsoid ARF shape is a consequence of the almost ellipsoid array geometry, with the ARF ellipse being approximately perpendicular to the major axis of the array shape (a long distance between stations means a greater 
time-delay and better slowness resolution). The ARFs for both AKASG and YKA (Figure $7 \mathrm{c}$ ) are sufficiently compact for high quality slowness estimates to be expected, although smoothing of the resulting slowness grids with the corresponding ARFs is recommended.

The MJAR array (Matsushiro, Japan, Figure $7 \mathrm{~d}$ ) is selected as an example of an intermediate aperture array $(10-20 \mathrm{~km})$. It is demonstrated by GibBons et al. (2008) that a plausible slowness vector estimate can be obtained for a regional P phase at MJAR, but it is clear from the beampacking grids that the broad ARF brings a significant risk of selecting a qualitatively incorrect slowness vector. The performance of MJAR has been examined in detail given that it is the only IMS array within $1000 \mathrm{~km}$ of the North Korea nuclear test site that recorded the explosions on both October 9, 2006, and May 25, 2009. Despite recording signals with a high SNR for both tests, MJAR failed to contribute to the automatic (SEL3 - the Standard Event List 3) event location estimate on both occasions. Given that subsequent offline efforts to estimate the direction uisng broadband f-k analysis fail to provide a qualitatively correct slowness estimate for either of these Pn phases, it is unsurprising that the fully automatic procedures fail. The beampacking grids in the top row of Figure 8 show clearly a pattern directed in the correct orientation towards the test site (at 306 degrees backazimuth) although local off-center maxima have led to a significant deviation in the reported backazimuth from the great-circle value. The lower panels, in which the slowness grids have been smoothed with respect to the ARF displayed in Figure 7 d), show a clear improvement with close-to-center local maxima which correspond well with the theoretical backazimuth and apparent velocity. Figure 8 indicates that the smooth functions resulting from the convolution of the observed and ARF slowness grids will provide robust slowness estimates for arrays of this aperture.

Of the 10-20 km aperture arrays (MJAR, ESDC, ILAR, KSRS, CMAR, ASAR, and EKA) there are great differences in the ability to process relatively high frequency signals. For example, MJAR and KSRS have similar apertures with MJAR having many smaller inter-site spacings than KSRS. However, whereas KSRS can often report stable and accurate slowness estimates of regional phases in the $2-4 \mathrm{~Hz}$ band, f-k estimates on MJAR at all but the very lowest frequencies are made impossible by the very complicated geology at that site (see KATO et al., 2005). On MJAR, the spectrogram beampacking with smoothing procedure displayed in Figure 8 is essentially the only strategy available for high frequency regional phases. On KSRS, qualitatively correct incoherent 
slowness estimates using the same procedure are possible, but the accuracy of coherent estimates (using classical $\mathrm{f}-\mathrm{k}$ analysis) in the $2-4 \mathrm{~Hz}$ band is likely to be better. In operational pipelines, it may be a good strategy to run both coherent and incoherent operations in parallel to provide two detection lists which can be compared and cross-validated.

Figure 9 displays filtered waveforms from a low-magnitude industrial seismic event on the Korean peninsula recorded by the KSRS array. The $2-4 \mathrm{~Hz}$ band is the highest frequency band for which the KSRS geometry will support coherent array processing but it is clear that the SNR in this band is extremely low, in particular for the P-arrival. The SNR in the 4-8 $\mathrm{Hz}$ band is better and beamforming in this band does manage to improve the SNR considerably. However, the beam of transformed spectrograms utilizes energy over a far wider spectrum and results in significant detections for both $\mathrm{P}$ and $\mathrm{S}$ phases on the scalar traces formed from averaging the spectrogram beams over frequency. Of interest for pipeline detection and estimation procedures is the property that the local maxima of the spectrogram beam scalars are very close to the signal onset time (this is confirmed by Song et al., 2010, for signals from microseismic events). The quality of these fully automatic arrival time estimates may reduce the need to apply repicking algorithms.

The 4 second ARF for the BRTR array in Turkey is displayed in Figure 7 e). The fact that such an ARF is unlikely even to be able to identify the quadrant of slowness space to which the true backazimuth and apparent velocity belong suggests that we have now reached the limit in array aperture over which we can apply the spectrogram beampacking procedure for parameter estimation. We note however that spectrogram beamforming is still a valid method for signal detection. The scan statistics algorithm of TAYLOR et al. (2010) is described for a single channel; the beamforming of spectrograms over adjacent sensors is likely only to reduce the variance of the background noise allowing a lower detection threshold.

The final panel of Figure 7, (f), displays a 4 second ARF for the medium period BRMAR array near Ankara; a large aperture (40 km) circular array in close proximity to BRTR (see KuleLI et al., 2001). The procedure described here can not be used on this array since the instrumentation does not record high frequency signals (the sampling interval is 0.25 seconds). However, this provides an illustration of the resolution in slowness that would be achieved using broadband or short period sensor deployments at that aperture. It is noted that panel (f) of Figure 7 is the only configuration 
for which sidelobes appear, also due to the array geometry.

\section{Conclusions and Discussion}

The diversity of the IMS seismic arrays leads to highly varying performance for seismic arrivals with different characteristics. Small aperture arrays provide good array processing for high frequency regional phases at the expense of array-gain and accuracy in parameter estimates for low frequency teleseismic phases. Large aperture arrays provide good teleseismic detection and characterization at the expense of coherence for high frequency regional phases. The inclusion of the so-called legacy seismic arrays into the IMS was very necessary as many of these arrays are the only stations to have existing records of many historical nuclear explosions. Waveforms from several of the legacy stations for many nuclear test explosions are openly available on the http://www.rdss.info/database/nedb/nedb_ent.html website (see BENNETT et al., 2010).

We have demonstrated that the spectrogram beamforming procedure of GiBBONs et al. (2008) for detecting and characterizing high frequency regional seismic phases is applicable to the IMS seismic arrays exceeding about $10 \mathrm{~km}$ aperture. The quality of the slowness estimates is improved significantly by smoothing the spectrogram beampacking grids using array response functions (ARFs) for signals with a period of 4 or 5 seconds. The smoothing procedure is deemed to be essential for the arrays with aperture under $20 \mathrm{~km}$. The stability of the procedure (with smoothing applied) is demonstrated in Figure 8 for the Pn arrivals at MJAR in Japan for the North Korea nuclear tests in 2006 and 2009. In many cases coherent processing may be applicable, and provide a greater accuracy than the incoherent procedure, but the f-k spectra may be subject to sidelobes due to spatial aliassing. The lower resolution estimates obtained from the smoothed spectrogram beampacking grids may help to identify the correct local maximum in the f-k spectra, in effect increasing the applicability of coherent array processing.

The detection part of the procedure is applicable to all IMS arrays regardless of aperture. There may be improvements to be made in the detection capability by applying algorithms from image processing (see TAYLOR et al., 2010) to the beams of transformed spectrograms and this may offer complementary detection lists which provide a very low false alarm rate for high frequency signals. The spectrogram beamforming method in effect measures a group velocity rather than a phase 
velocity and the detection threshold will always be higher for this procedure than for arrays where coherent processing is applied. Experience in operating the algorithm of GiBBONs et al. (2008) on data from the NOA array (since 2005) indicates that an SNR of about 2.0 on waveforms from individual channels is necessary for the incoherent system to work well. The classification of all detected signals is essential whether or not the signals in question are of interest to the monitoring aims. For example, many false teleseismic detections at NOA and other large aperture arrays are high frequency regional signals that have been classified incorrectly in the low frequency band used for teleseismic processing. Application of the spectrogram beamforming algorithm may classify these phases correctly as regional arrivals such that they can be screened out of the teleseismic detection lists, where they are likely to contribute to spurious event hypotheses.

The measure with which we assess any modification to the pipeline detection algorithms has to be the quality of the resulting automatic seismic event bulletins. This comprises completeness (how many real events are well represented in the automatic bulletin), the false event rate (how many entries in the automatic bulletin do not correspond to real events), and the location accuracy (what additional effort will the analyst require to generate a reviewed location estimate). The left panel of Figure 10 shows all of the REB events in a region of northern Europe over a one year time interval for which the location was constrained using a phase pick from the large aperture NOA array. The dark symbols the subset of these events which were also associated with a fully automatic NOA phase detection in the SEL3 bulletin. The dark symbols account for very few of the reviewed events, meaning that significant analyst effort has been required to add phases from NOA. The right panel of Figure 10 shows events for the same region and time interval from the NORSAR Regional Event Bulletin, the dark symbols indicating events with fully automatic phase associations from each of the 3 primary seismic IMS arrays NOA, ARCES, and FINES. The spectrogram beamforming procedure operates on NOA array data at NORSAR but not at the IDC, and the bulletins are not entirely comparable as NORSAR is free to use non-IMS seismic stations that are not available to the IDC. However, there are clearly far more events for which the automatic location estimate is controlled by all 3 primary seismic arrays when the incoherent processing algorithm is applied to NOA data. Most of the events in southern Norway with pale symbols in the right panel of Figure 10 are constrained with both regional $\mathrm{P}$ and $\mathrm{S}$ automatic phase detections at NOA; it is the ARCES array for which 
detections are missing for most such events.

It is not necessarily the case that, from a resources point of view, it is desirable to increase the number of events as displayed in Figure 10. However, it is necessary to point out that it is only the absence of an algorithm able to classify high frequency regional phases over the NOA aperture which prevents all of the 3 -primary station events in the right panel from appearing in the left panel. HicKs et al. (2001) pointed out that the number of events in Norway in the REB would be smaller than for the NORSAR event bulletin given that the small aperture NORES array (co-located with part of the NOA array) was contributing to the NORSAR detection lists at the time. Were NORES an IMS array instead of NOA, the number of 3-primary station events would be far greater than indicated in the right panel of Figure 10 given that NORES would also have a lower detection threshold than NOA using incoherent methods.

The importance of high frequency seismic energy in nuclear explosion monitoring must not be underestimated. The propagation of seismic energy well in excess of $10 \mathrm{~Hz}$ has been observed over distances in excess of $1000 \mathrm{~km}$ (e.g. Bowers et al., 2001) and it is imperative that we obtain a global overview of the degree to which different paths attenuate high frequency energy. It was demonstrated in Figure 2 how weaker events result in a lower signal amplitude over a wide range of frequencies which, due to the spectral level of the background noise, results in better SNR at the higher frequencies. It is precisely the lower frequency energy which is likely to diminish most given a lower explosion yield (compare, for example, the spectra from the 2006 and higher yield 2009 underground nuclear tests at the North Korea test site; Gibbons and Ringdal, 2012). The same is true of the cavity-decoupling evasion scenario (see Stevens et al., 2006), with explosions with lower charge density resulting in regional seismic signals with far less low frequency energy.

The IMS stations at which high-frequency energy arrivals are observed, but which cannot be processed due to unfavourable array geometries, need to be identified. In situations where events are repeating, or where high quality calibration information is available, the empirical matched field processing method of HARRIS and KVAERnA (2010) may be able to exploit phase information associated with high frequency energy above the limit where classical array processing can be applied. For each instance where signals are being detected by IMS arrays that are not correctly classified, we need to examine the reason. The incorrect classification of a detected phase may have as serious 
consequences as a missed phase given that an automatic association algorithm has to process the output from the detection streams at each station. Applying the algorithms described here may reduce significantly the number of phases which are incorrectly characterized and may allow the remaining detections to be filtered out for subsequent manual analysis.

\section{Acknowledgements}

Data from IMS array stations were obtained via the IDC in Vienna. I am grateful for access to data from additional stations of the Geoscience Australia (AU) network used in this study which were obtained via the IRIS Data Management Center. I am also grateful for the constructive comments of two anonymous referees.

Maps were created using GMT software (Wessel and Smith, 1995).

\section{References}

Bennett, T. J., Oancea, V., Barker, B. W., Kung, Y. L., Bahavar, M., et al. (2010), The Nuclear Explosion Database (NEDB): A New Database and Web Site for Accessing Nuclear Explosion Source Information and Waveforms. Seismological Research Letters 81, 12-25, doi: 10.1785/gssrl.81.1.12.

Bowers, D., Marshall, P. D., and Douglas, A. (2001), The level of deterrence provided by data from the SPITS seismometer array to possible violations of the Comprehensive Test-Ban in the Novaya Zemlya region. Geophys. J. Int. 146, 425-438, doi:10.1046/j.1365-246x.2001.01462.x.

Bungum, H., and Husebye, E. S. (1971), Errors in time delay measurements. Pure appl. geophys. $91,56-70$.

Capilla, C. (2006), Application of the Haar wavelet transform to detect microseismic signal arrivals. Journal of Applied Geophysics 59, 36-46, doi:10.1016/j.jappgeo.2005.07.005.

Christie, D. R., and Campus, P., The IMS Infrasound Network: Design and Establishment of Infrasound Stations, in Infrasound Monitoring for Atmospheric Studies (eds. A. Le Pichon, 
E. Blanc, and A. Hauchecorne) (Springer Science + Business Media B.V., Dordrecht, Netherlands 2010), chap. 2, pp. 29-75. ISBN 978-1-4020-9507-8.

Dahlman, O., Mykkeltveit, S., and HaAk, H., Nuclear Test Ban: Converting Political Visions to Reality (Springer Media B.V., Dordrecht, The Netherlands 2009). ISBN 978-1-4020-6883-6.

Douglas, A., Seismometer Arrays - Their Use in Earthquake and Test Ban Seismology, in International Handbook of Earthquake and Engineering Seismology (eds. W. H. K. Lee, H. Kanamori, P. C. Jennings, and C. Kisslinger) (Academic Press 2002), pp. 357-367.

Gendron, P., Ebel, J., and Manolakis, D. (2000), Rapid Joint Detection and Classification with Wavelet Bases via Bayes Theorem. Bull Seism Soc Am 90, 764-774.

Gibbons, S. J., and Ringdal, F. (2012), Seismic Monitoring of the North Korea Nuclear Test Site Using a Multi-Channel Correlation Detector. IEEE Trans. Geosci. Remote Sensing In press.

Gibbons, S. J., Ringdal, F., and Kværna, T. (2008), Detection and characterization of seismic phases using continuous spectral estimation on incoherent and partially coherent arrays. Geophysical Journal International 172, 405-421, doi:10.1111/j.1365-246X.2007.03650.x.

HëGe, M., and Joswig, M. (2009), Spatiotemporal characterization of interswarm period seismicity in the focal area Nový Kostel (West Bohemia/Vogtland) by a short-term microseismic study. Geophysical Journal International 179, 1071-1079, doi:10.1111/j.1365-246X.2009.04320.x.

Harris, D. B., and Kvaerna, T. (2010), Superresolution with seismic arrays using empirical matched field processing. Geophysical Journal International 182, 1455-1477, doi:10.1111/j.1365246X.2010.04684.x.

Hicks, E. C., Bungum, H., and Ringdal, F. (2001), Earthquake Location Accuracies in Norway Based on a Comparison between Local and Regional Networks. Pure and Applied Geophysics 158, 129-141, doi:10.1007/PL00001152.

Joswig, M. (1990), Pattern recognition for earthquake detection. Bull. Seism. Soc. Am. 80, 170-186.

Joswig, M. (1995), Automated classification of local earthquake data in the BUG small array. Geophysical Journal International 120, 262-286, doi:10.1111/j.1365-246X.1995.tb01818.x. 
Kato, M., Nakanishi, I., and Takayama, H. (2005), Variation of teleseismic short-period waveforms at Matsushiro Seismic Array System. Earth, Planets, Space 57, 563-570.

Kennett, B. L. N., The Seismic Wavefield. Volume II: Interpretation of Seismograms on Regional and Global Scales (Cambridge University Press, Cambridge, United Kingdom 2002). ISBN 0-52100665-1.

Kennett, B. L. N., Brown, D. J., Sambridge, M., and Tarlowski, C. (2003), Signal Parameter Estimation for Sparse Arrays. Bulletin of the Seismological Society of America 93, 1765-1772.

Kuleli, S., Zor, E., Türkelli, N., Sandvol, E., Seber, D., et al. (2001), The IMS Belbasi Seismic Array (BRAR) in Central Turkey. Seismological Research Letters 72, 60-69.

Kværna, T., and RIngdal, F. (1992), Integrated array and three-component processing using a seismic microarray. Bull. Seism. Soc. Am. 82, 870-882.

Kverna, T., Ringdal, F., and Given, J., Application of Detection Probabilities in the IDC Global Phase Association Process, in Proceedings of the 2011 Monitoring Research Review, Tucson, Arizona, September 13-15, 2011. Ground-based Nuclear Explosion Monitoring Technologies, Report LA-UR-11-04823 (2011), pp. 302-312.

Levander, A., and Nolet, G., Perspectives on Array Seismology and USArray, in Seismic Earth: Array Analysis of Broadband Seismograms (eds. A. Levander and G. Nolet) (American Geophysical Union 2005), pp. 1-6. Geophysical Monograph 157.

Mykkeltveit, S., Ringdal, F., Kværna, T., and Alewine, R. W. (1990), Application of regional arrays in seismic verification research. Bull Seism Soc Am 80, 1777-1800.

Nishida, K., Kawakatsu, H., FukaO, Y., and Obara, K. (2008), Background Love and Rayleigh waves simultaneously generated at the Pacific Ocean floors. Geophysical Research Letters 35, L16307+, doi:10.1029/2008GL034753.

Oonincx, P. J. (1999), A wavelet method for detecting S-waves in seismic data. Computational Geosciences 3, 111-134, doi:10.1023/A:1011527009040. 
Picozzi, M., Parolai, S., and Bindi, D. (2010), Deblurring of frequency-wavenumber images from small-scale seismic arrays. Geophys. J. Int. 181, 357-368, doi:10.1111/j.1365-246X.2009.04471.x.

Prieto, G. A., Parker, R. L., and Vernon, F. L. (2009), A Fortran 90 library for multitaper spectrum analysis. Computers and Geosciences 35, 1701-1710.

Ringdal, F., and Husebye, E. S. (1982), Application of Arrays in the Detection, Location, and Identification of Seismic Events. Bull. Seism. Soc. Am. 72, S201-S224.

Ringdal, F., and Kverna, T. (1989), A multi-channel processing approach to real time network detection, phase association, and threshold monitoring. Bull. seism. Soc. Am. 79, 1927-1940.

Ringdal, F., Husebye, E. S., and Dahle, A., P-Wave Envelope Representation in Event Detection Using Array Data, in Exploitation of Seismograph Networks (ed. K. G. Beauchamp), no. 11 in Series E: Applied Sciences (Noordhoff - Leiden 1975), pp. 353-372.

Romney, C. F., VELA Overview: The Early Years of the Seismic Research Program, in The VELA Program: A Twenty-Five Year Review of Basic Research (ed. A. U. Kerr) (1985), pp. 38-65.

Rost, S., and Thomas, C. (2002), Array Seismology: Methods and Applications. Rev. Geophys. 40. 1008, doi:10.1029/2000RG000100.

Rost, S., and Thomas, C. (2009), Improving Seismic Resolution Through Array Processing Techniques. Surveys in Geophysics 30, 271-299, doi:10.1007/s10712-009-9070-6.

Schweitzer, J., Fyen, J., Mykneltveit, S., and Kverna, T., Chapter 9: Seismic Arrays, in IASPEI New Manual of Seismological Observatory Practice (ed. P. Bormann) (GeoForschungsZentrum, Potsdam 2002). 52 pp.

Selby, N. D. (2011), Improved Teleseismic Signal Detection at Small-Aperture Arrays. Bull. Seism. Soc. Am. 101, 1563-1575, doi:10.1785/0120100253.

Song, F., Kuleli, H. S., Toksoz, M. N., Ay, E., and Zhang, H. (2010), An improved method for hydrofracture-induced microseismic event detection and phase picking. Geophysics 75, A47-52, doi:10.1190/1.3484716. 
Stevens, J. L., Gibbons, S., Rimer, N., Xu, H., Lindholm, C., et al. (2006), Analysis and simulation of chemical explosions in nonspherical cavities in granite. Journal of Geophysical Research - Solid Earth 111, B04306+, doi:10.1029/2005JB003768.

Taylor, S. R., Arrowsmith, S. J., and Anderson, D. N. (2010), Detection of Short Time Transients from Spectrograms Using Scan Statistics. Bull. Seism. Soc. Am. 100, 1940-1951, doi: $10.1785 / 0120100017$.

Thomson, D. J. (1982), Spectrum estimation and harmonic analysis. Proc. IEEE 70, 1055-1096.

Wessel, P., and Smith, W. H. F. (1995), New version of the Generic Mapping Tools. EOS Trans. Am. Geophys. Union 76, 329.

Withers, M., Aster, R., Young, C., Beiriger, J., Harris, M., et al. (1998), A comparison of select trigger algorithms for automated global seismic phase and event detection. Bull. Seism. Soc. Am. 88, 95-106.

Yomogida, K. (1994), Detection of anomalous seismic phases by the wavelet transform. Geophysical Journal International 116, 119-130, doi:10.1111/j.1365-246X.1994.tb02131.x.

Zhang, H., Thurber, C., and Rowe, C. (2003), Automatic P-Wave Arrival Detection and Picking with Multiscale Wavelet Analysis for Single-Component Recordings. Bull. Seism. Soc. Am. 93, 1904-1912, doi:10.1785/0120020241. 


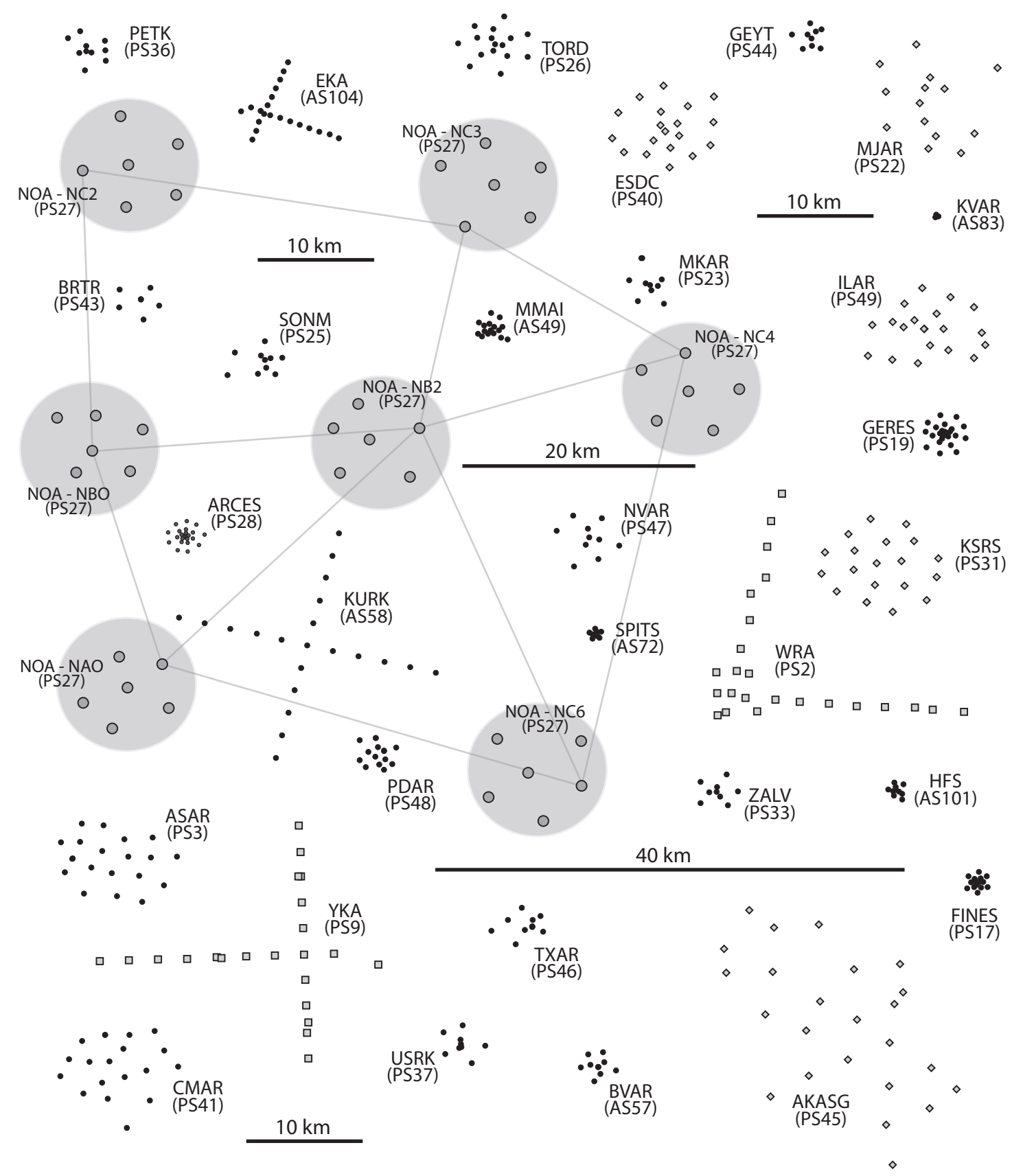

Figure 1: IMS array stations as of September 2011 drawn to a common scale. The seven subarrays of the large aperture NORSAR array (NOA) are shaded and labelled in parentheses. The 3-C broadband sensors in each subarray are linked by grey lines. The so-called medium period arrays are not displayed. 

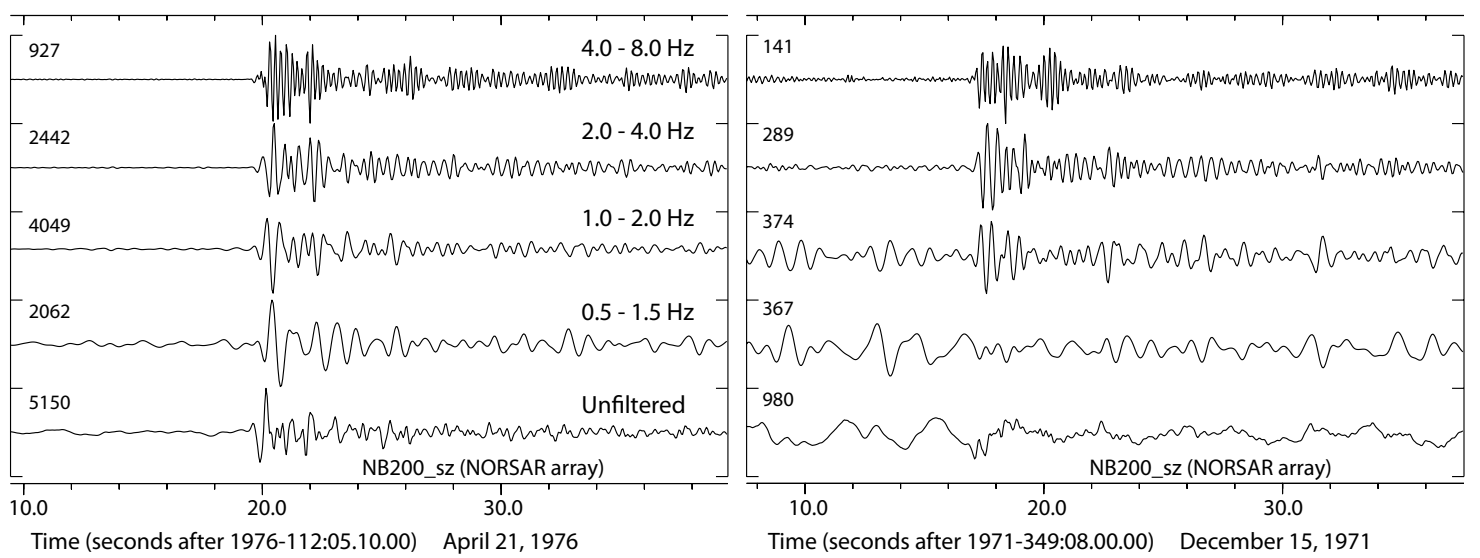

Figure 2: Signals from two explosions at the Semipalatinsk Test Site (STS) in the former Soviet Union recorded on site NB200 of the NORSAR array, raw waveforms and bandpass filtered as indicated. 

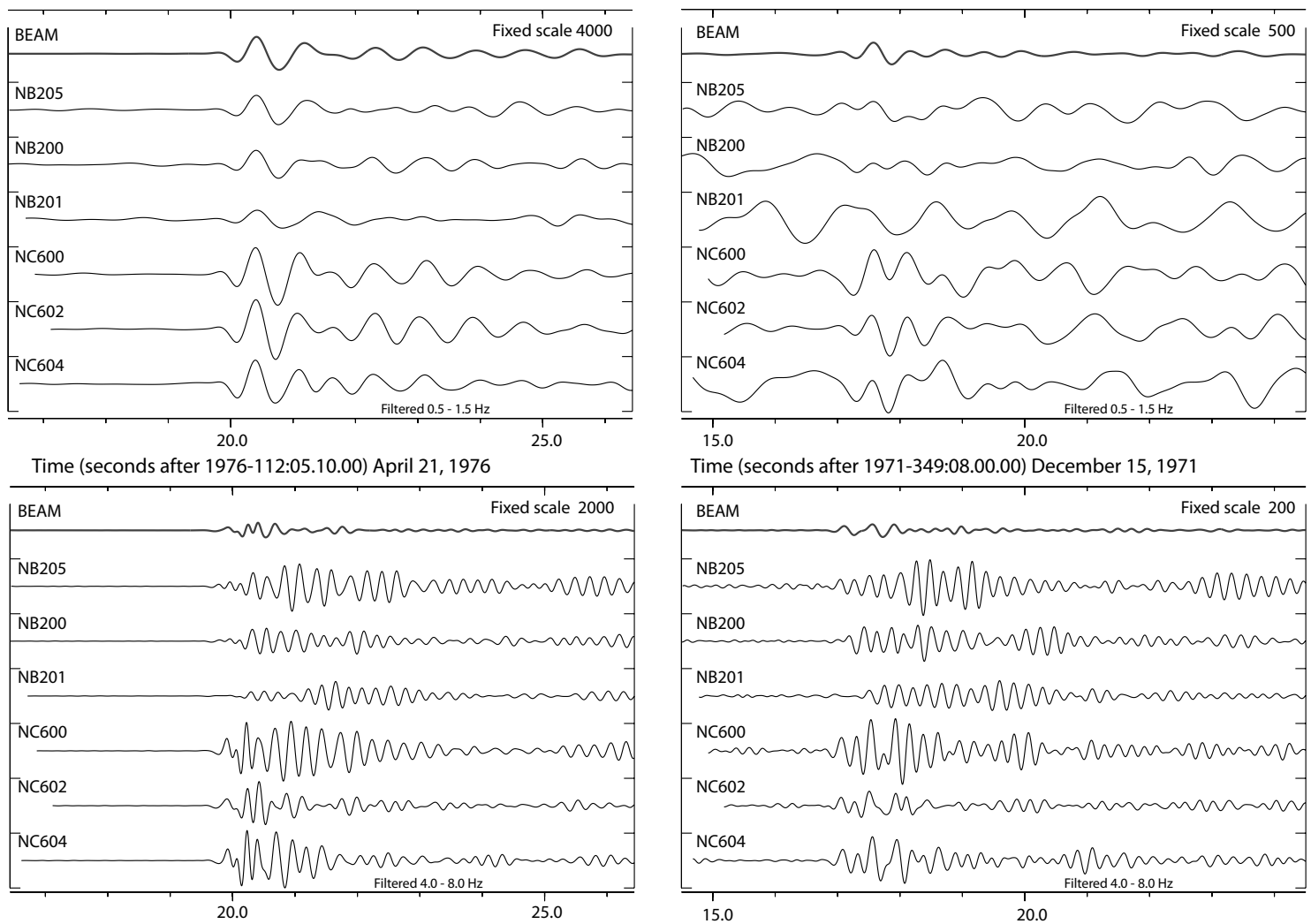

Time (seconds after 1971-349:08.00.00) December 15, 1971

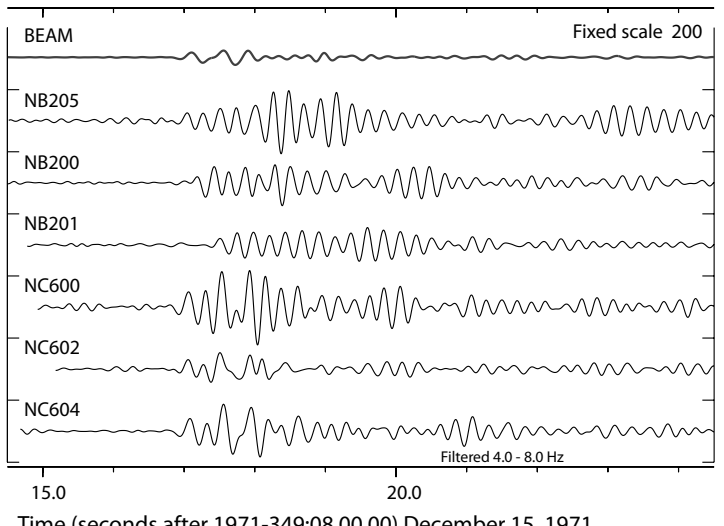

Time (seconds after 1976-112:05.10.00) April 21, 1976

Figure 3: Signals from the two events displayed in Figure 2 on the channels displayed aligned according to optimal time-shifts. The beams are formed from all 42 sites that comprise the current NORSAR array (PS27). The waveforms in the upper and lower panels are bandpass filtered 0.5-1.5

$\mathrm{Hz}$ and 4.0-8.0 $\mathrm{Hz}$ respectively. 

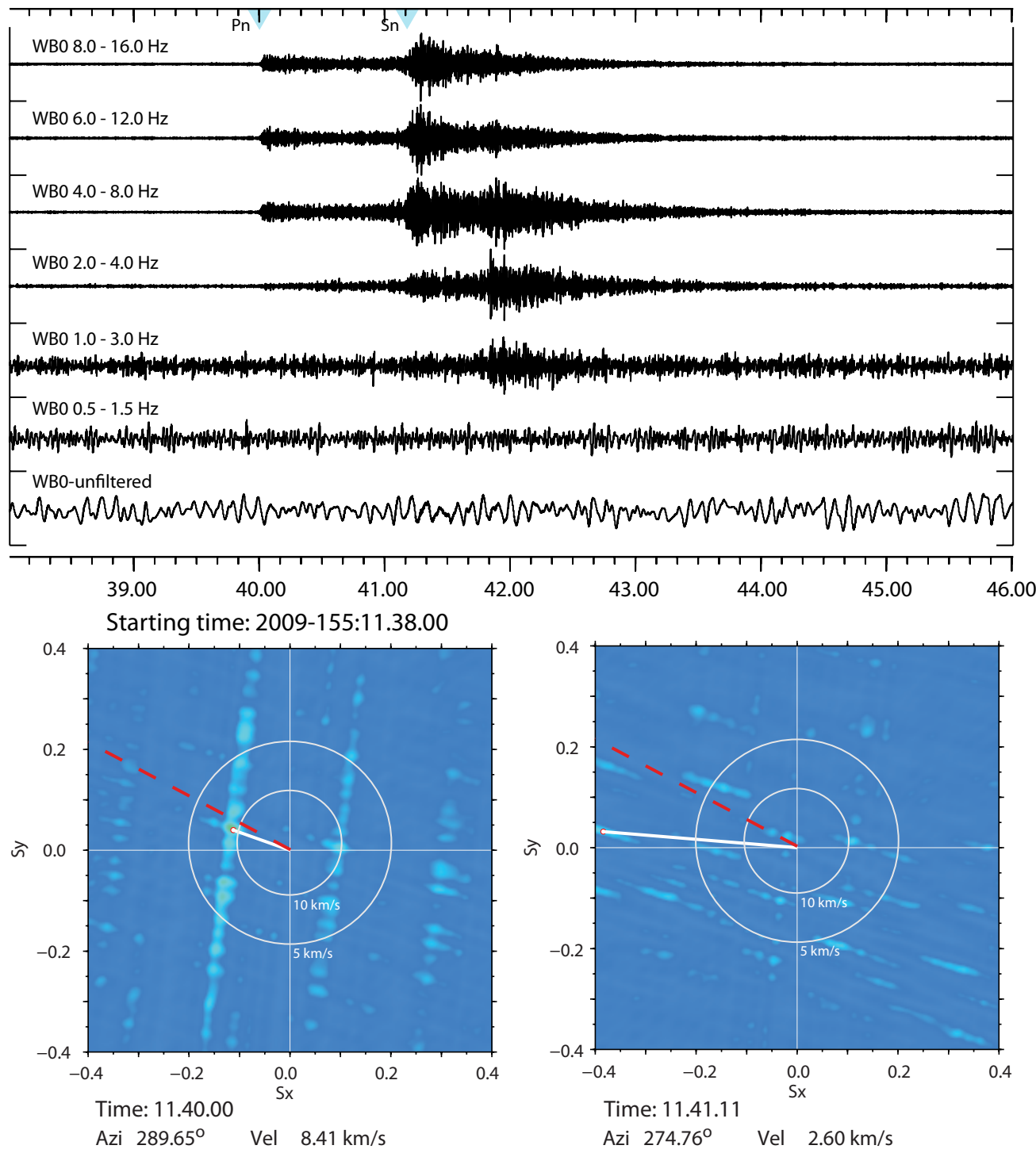

Figure 4: A regional event in the IDC Reviewed Event Bulletin (REB) observed on the Warramunga array (WRA) in Australia June 4, 2009, together with relative-beam-power $\left(P_{\text {rel }}\right)$ slowness grids for Pn and Sn arrivals using broadband f-k analysis in the $2-4 \mathrm{~Hz}$ band. The horizontal slownesses $s_{x}$ and $s_{y}$ resulting in the highest $P_{\text {rel }}$ are found and then $v_{\text {app }}=\left(s_{x}^{2}+s_{y}^{2}\right)^{-1 / 2}$ and BAZ $=\operatorname{atan} 2\left(s_{x}, s_{y}\right)$ are calculated. The dashed red lines indicate a backazimuth of 298 degrees towards an origin at $16.78^{\circ} \mathrm{S}$ $128.23^{\circ} \mathrm{E}, 6.6$ degrees from WRA, an epicenter determined using regional $\mathrm{P}$ and $\mathrm{S}$ phases on stations of the AU network operated by Geoscience Australia. The rings indicate typical boundaries between teleseismic P-phases ( $v_{\mathrm{app}}>10 \mathrm{~km} / \mathrm{s}$, near the center), regional P-phases (between the rings), and regional S-phases (beyond the outer ring).

Draft

Pure appl. Geophys. 

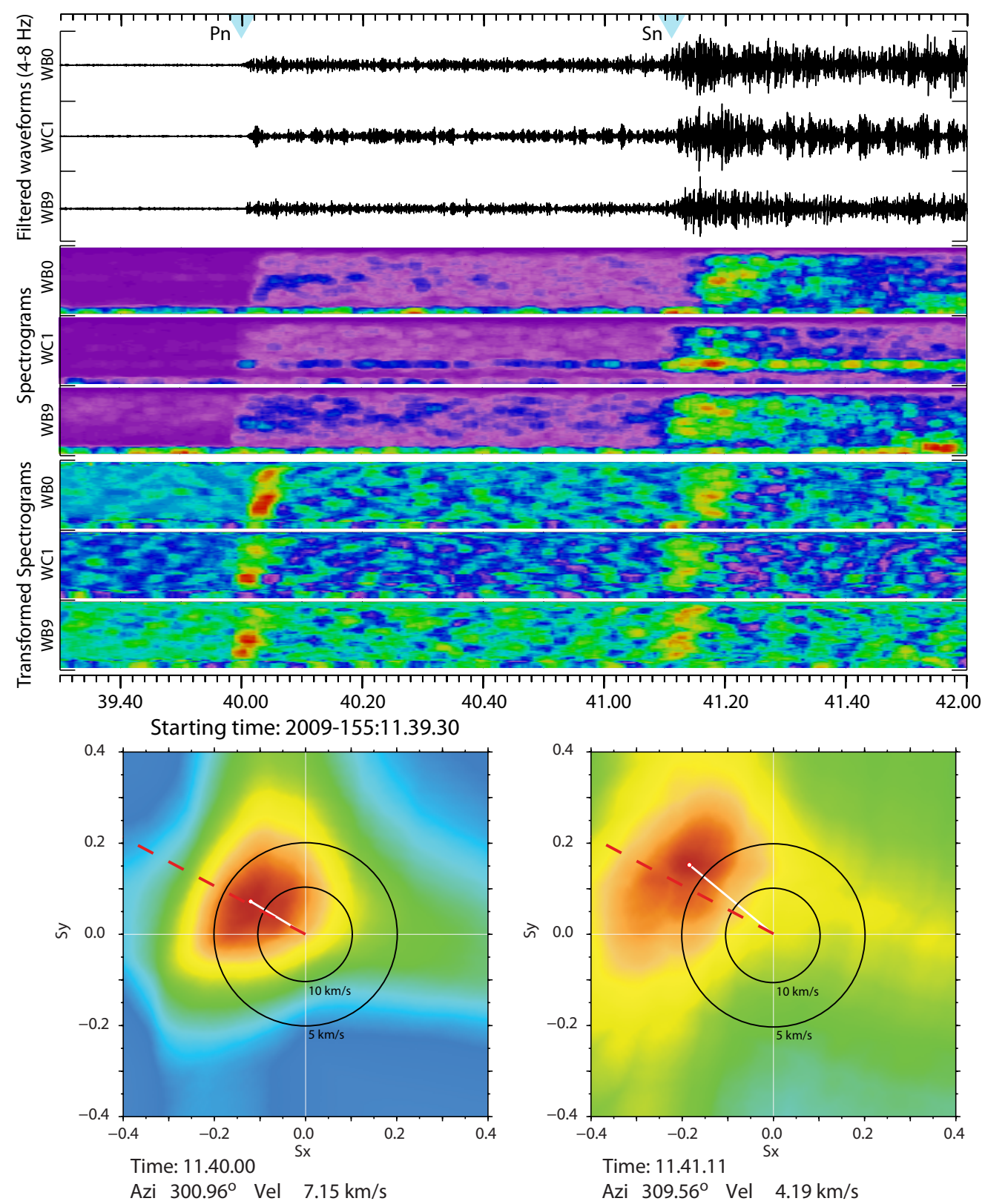

Figure 5: Estimation of slowness vectors using spectrogram beamforming for the Warramunga Pn and Sn arrivals displayed in Figure 4. The vertical scale for each of the spectrogram panels goes from 0 to $20 \mathrm{~Hz}$, the Nyquist frequency. The slowness grids display the maximum value of the spectrogram beams, averaged over all frequencies, and will be referred to as beampacking grids. The dashed lines indicate a backazimuth of 298 degrees. 


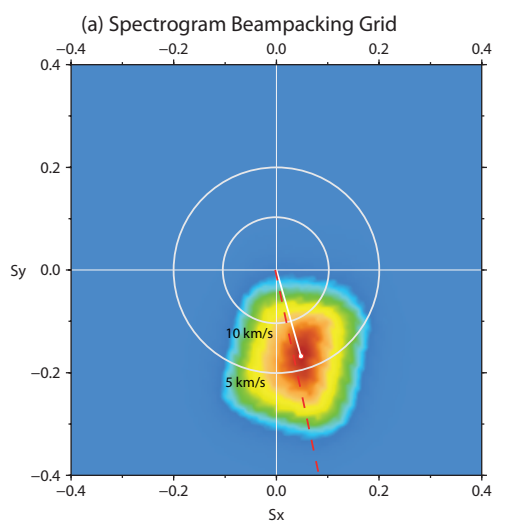

Azi $164.05^{\circ}$ Vel $5.72 \mathrm{~km} / \mathrm{s}$
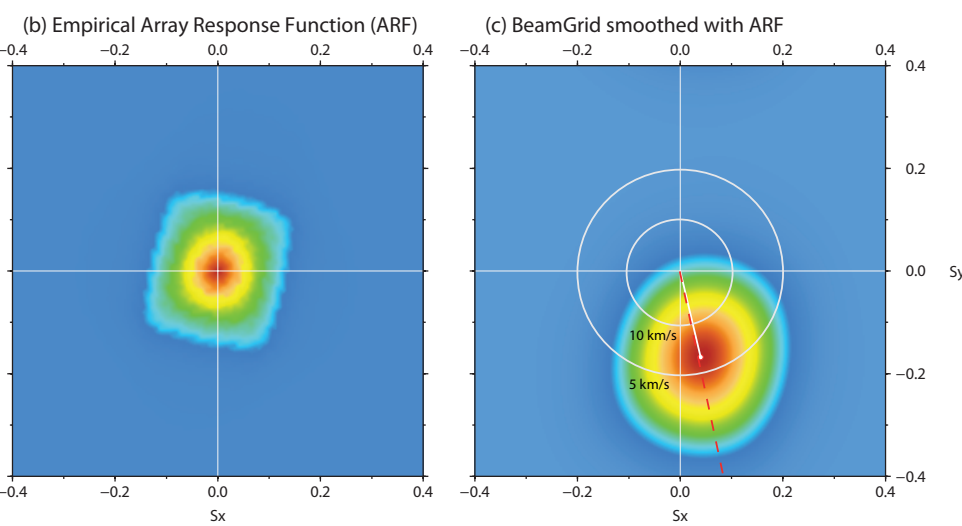

Azi $166.61^{\circ}$ Vel $5.79 \mathrm{~km} / \mathrm{s}$

Figure 6: Smoothing (right) of a beampacking slowness grid (left) with an empirical array response function (ARF, center) for the Kurchatov array, KURK, in Kazakhstan. The empirical ARF is calculated by copying the transformed spectrogram from one element to all sensors of the array and then subjecting these identical patterns to the same beamforming procedure used to form the beampacking slowness grid on the left. The reference time point for the calculation is 2009332:07.20.46 and corresponds to a first regional P-wave arrival from a routine quarrying blast at the Kara Zhyra mine at a distance of approximately 0.68 degrees. The coordinates of the blast are $50.02^{\circ} \mathrm{N}, 78.73^{\circ} \mathrm{E}$ and the receiver-to-source backazimuth of 168 degrees is indicated by the dashed lines. 

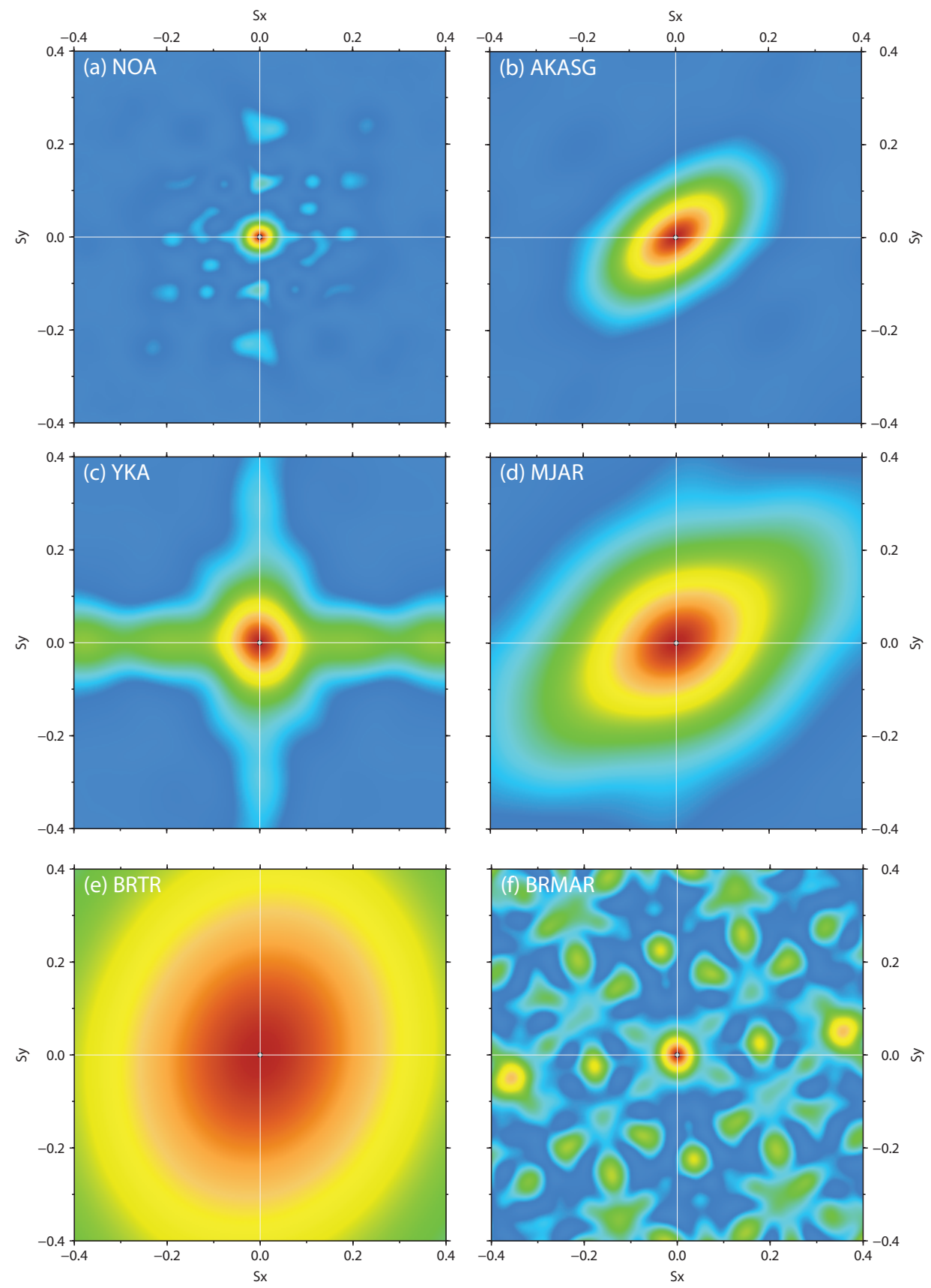

Figure 7: Array response functions for a 4 second period signal on the seismic arrays as indicated. The geometries of all arrays are provided in Figure 1 except for BRMAR, a medium period array with an aperture of approximately $30 \mathrm{~km}$ close to the BRTR short period array at Keskin, Turkey (KUlELI et al., 2001). 

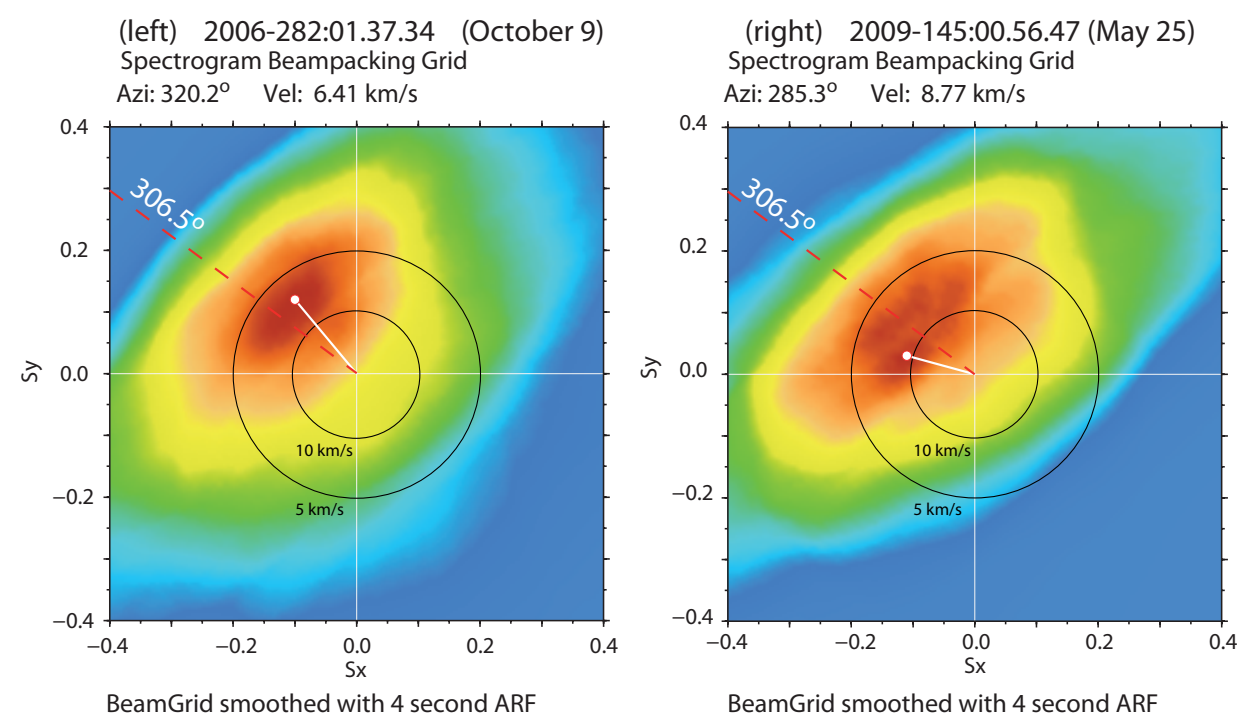

BeamGrid smoothed with 4 second ARF Azi: $302.0^{\circ} \quad$ Vel: $10.59 \mathrm{~km} / \mathrm{s}$
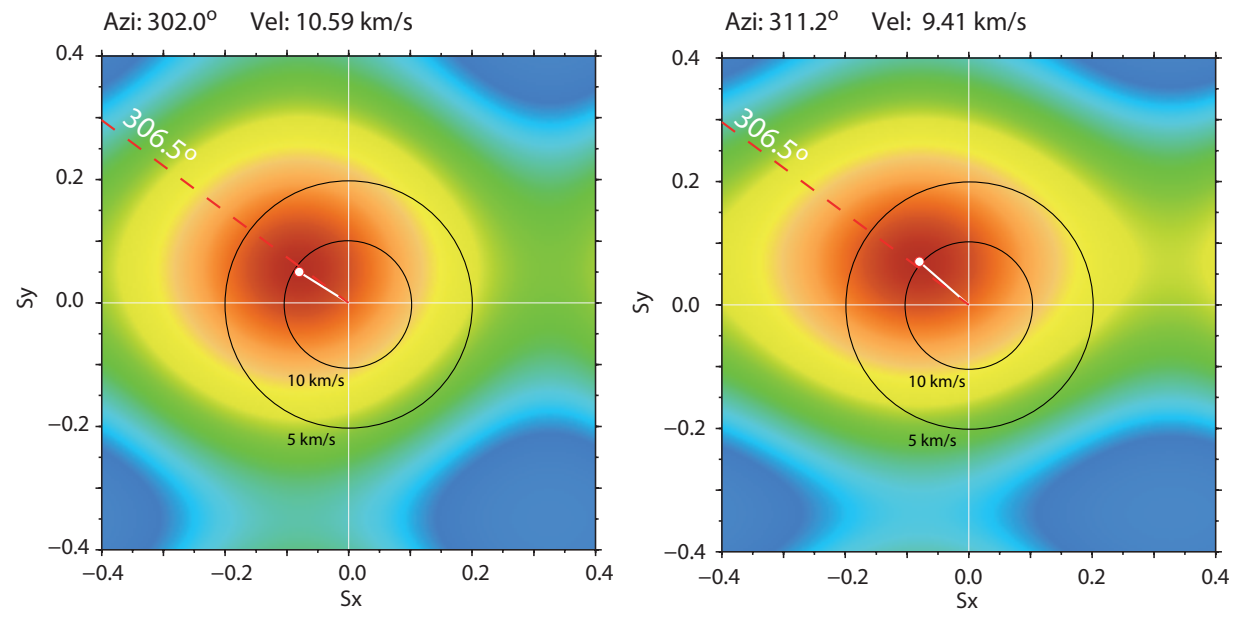

Figure 8: Slowness estimates for the Pn arrival at the MJAR array in Japan for the 2006 (left) and 2009 (right) North Korea nuclear tests at a distance of approximately $950 \mathrm{~km}$. The top panels show the beamgrid slowness maps simply constructed from delay-and-sum of the transformed spectrograms for the vertical channels of the array. The lower panels show the result of smoothing each of the above grids with the theoretical 4 second array response function for MJAR displayed in Figure 7. The dashed line in each panel indicates the backazimuth from the array to the nuclear test site. 


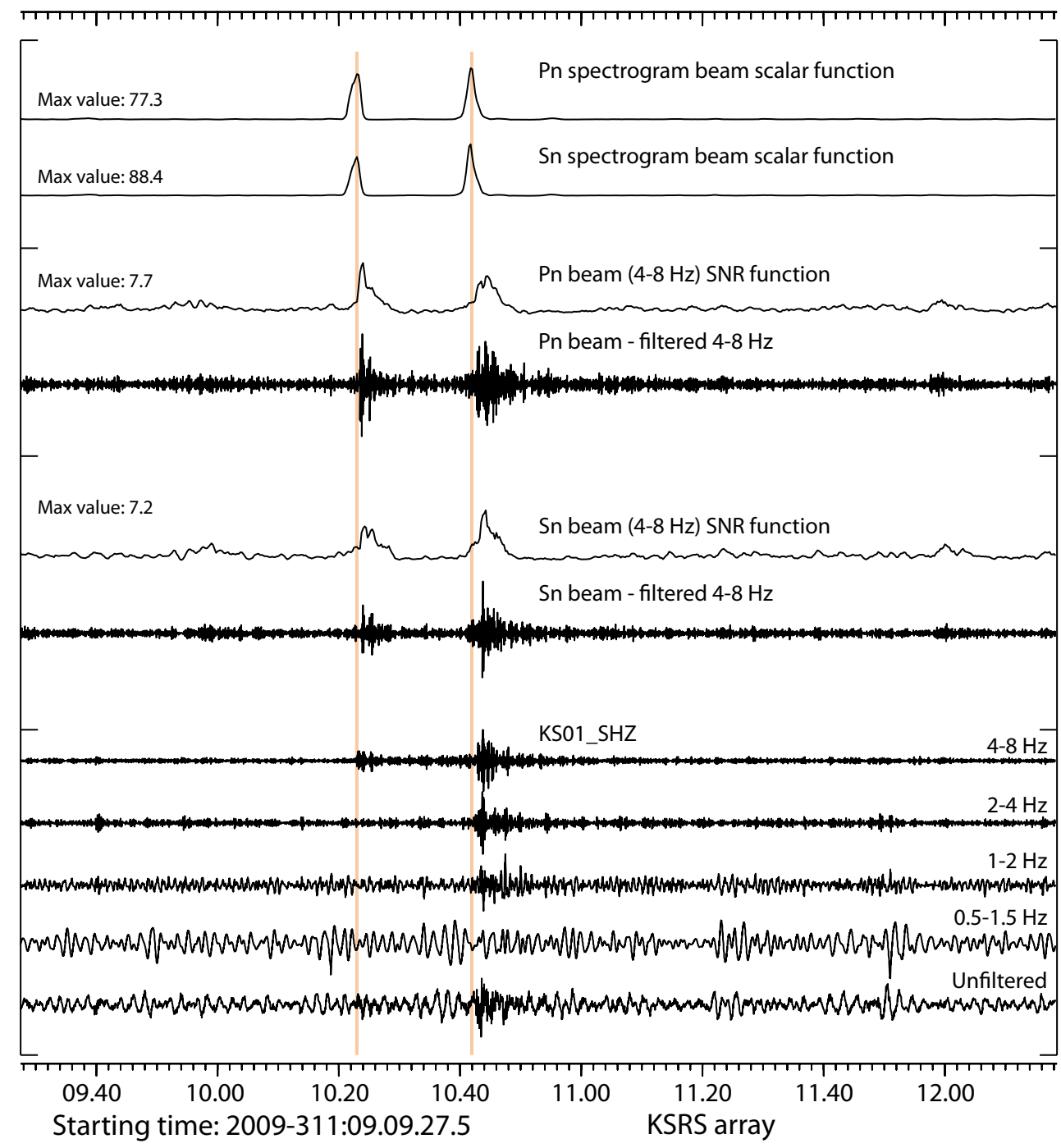

Figure 9: Signals on the KSRS array in the Republic of Korea from a quarry blast within a distance of 2 degrees. It is clear that the SNR is optimal in a frequency band higher than that amenable to coherent processing on this array. The scalar functions derived from the transformed spectrogram beams give the impression of greater significance to the $\mathrm{P}$ and $\mathrm{S}$ detection peaks than for the classical beam SNR functions. Note also that the local maxima of the SNR functions occur somewhat later than the local maxima of the spectrogram beam scalar functions. 

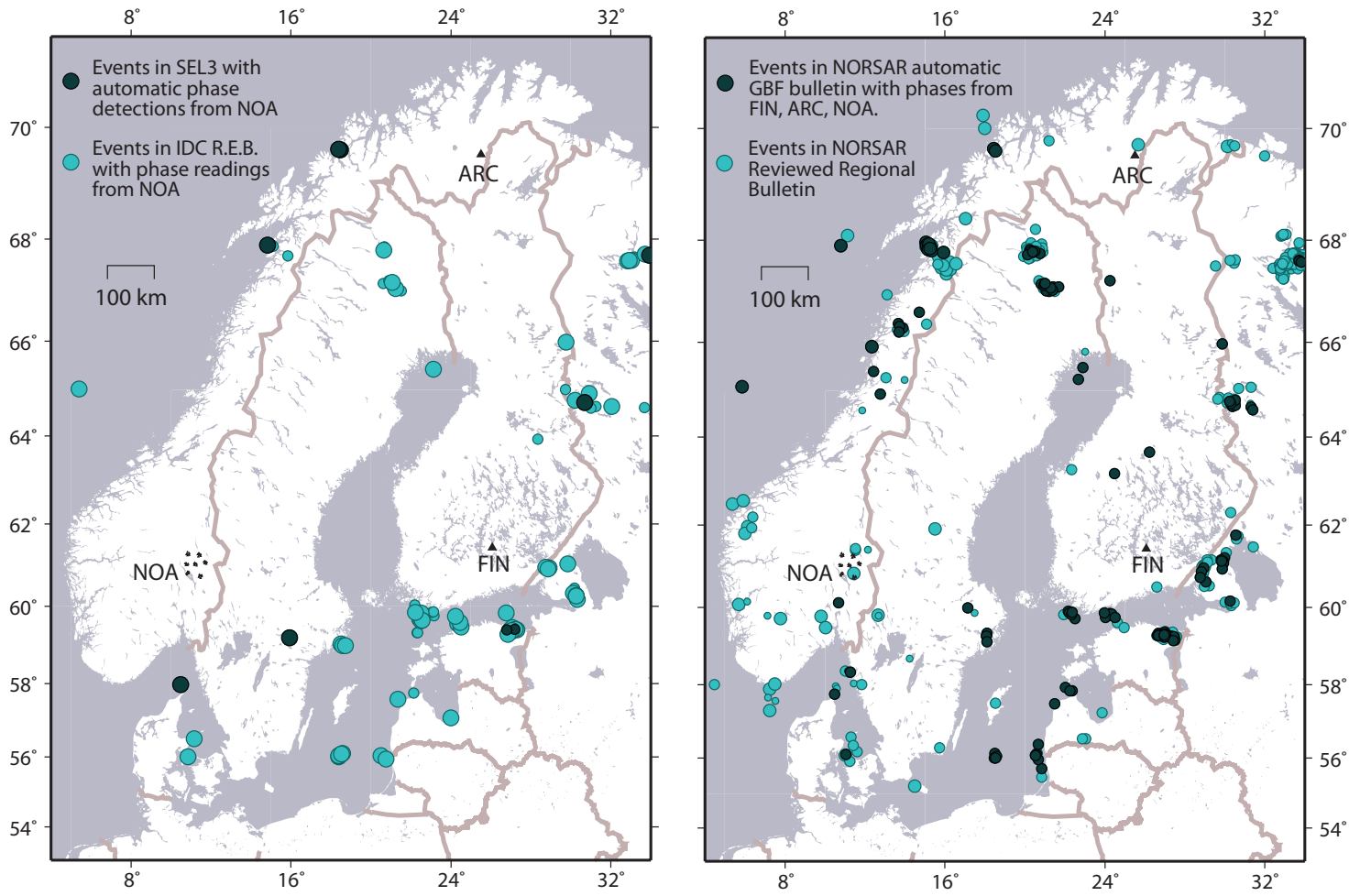

Figure 10: Events between 2007-078 and 2008-078 in the reviewed event bulletins (light blue symbols) for the IDC (left) and NORSAR (right). The IDC REB events (light blue, left panel) are restricted to events that include a phase reading from the large aperture NOA array. The dark symbols indicate events in the fully automatic bulletins at the same data centers which include at least one NOA phase detection. (Dark symbols in the right hand panel must also include phases from the primary IMS arrays FINES and ARCES.) At NORSAR, two parallel and independent systems process incoming data from the NOA array. A coherent process runs to detect and classify teleseismic phases and a process of spectrogram beamforming described by GiBBons et al. (2008) runs to detect and classify regional phases. The fully automatic GBF bulletin (RINGDAL and KVÆRnA, 1989) uses phase detections from the second of these processes. 